\title{
Clinical significance of preoperative chemoradiotherapy for advanced esophageal cancer, evaluated by propensity score matching and weighting of inverse probability of treatment
}

\author{
YOSHINORI FUJIWARA ${ }^{1}$, SHUICHI FUKUDA ${ }^{2}$, MASANORI TSUJIE ${ }^{2}$, KOTARO KITANI ${ }^{2}$, \\ MASAO YUKAWA ${ }^{2}$, MASATOSHI INOUE ${ }^{2}$, YUSAKU WATANABE ${ }^{1}$, MASAHARU HIGASHIDA $^{1}$, \\ HISAKO KUBOTA ${ }^{1}$, TOSHIMASA OKADA ${ }^{1}$, ATSUSHI TSURUTA ${ }^{1}$ and TOMIO UENO ${ }^{1}$ \\ ${ }^{1}$ Department of Digestive Surgery, Kawasaki Medical School, Kurashiki, Okayama 701-0192; ${ }^{2}$ Department of Surgery, \\ Nara Hospital, Kindai University, Ikoma, Nara 630-0293, Japan
}

Received June 14, 2018; Accepted March 29, 2019

DOI: $10.3892 / \mathrm{mco} .2019 .1843$

\begin{abstract}
The present study used inverse probability of treatment weighting (IPTW) and propensity score matching (PSM) to compare survival benefits among 112 patients with resectable, stage II-IV esophageal squamous cell carcinoma (SCC) treated between 1996 and 2016 with neoadjuvant chemoradiotherapy (NAC) plus surgery (Group A, $n=55$ ) or with surgery alone (Group B, $n=57$ ). Their propensity scores (PS) were calculated using a multivariable logistic regression model in which age, sex, cancer site, primary tumor length, cTNM stage, lymph node metastasis and depth of tumor invasion were the independent variables, and used to match Groups A and B according to the IPTW and matching method. After IPTW and PSM, univariate analysis was used to assess overall survival (OS) and disease-free survival (DFS), followed by Cox proportional hazard models for OS using IPTW between the two groups and the subgroups. After PSM, 5-year OS and DFS were significantly higher in Group A (OS: 65.2\%, DFS: 65.2\%) compared with Group B (OS: $31.2 \%$, DFS: $20.87 \%$ ). Similarly, after IPTW, OS and DFS were significantly higher in Group A compared with Group B patients. Five-year OS was 73.18\% for Group A and 37.69\% for Group B (hazard ratio: $0.2899,95 \%$ confidence interval: $0.1167-0.7205)$. To conclude,
\end{abstract}

Correspondence to: Dr Yoshinori Fujiwara, Department of Digestive Surgery, Kawasaki Medical School, 477 Matsushima, Kurashiki, Okayama 701-0192, Japan

E-mail: yyfujiwara@nifty.com; yyfujiwara@med.kawasaki-m.ac.jp

Abbreviations: $\mathrm{CF}$, fluorouracil and cisplatin regimen; $\mathrm{CP}$, cisplatin; CRT, chemoradiotherapy; DCF, docetaxel, cisplatin, and fluorouracil; DFS, disease-free survival; EC, esophageal cancer; IPTW, inverse probability of treatment weighting; OS, overall survival; PS, propensity score; PSM, propensity score matching; SCC, squamous cell carcinoma

Key words: esophageal cancer, chemoradiotherapy, esophagectomy, propensity score treatment was more effective in Group A patients with clinical stage II, N0 and T3 disease involving the mid-esophagus. It was concluded that for patients with esophageal SCC, NAC plus esophagectomy exhibited improved survival compared with surgery alone, as demonstrated by use of IPTW and PSM methods.

\section{Introduction}

Esophageal cancer (EC) is the eighth most common cancer worldwide, with an estimated 455,784 new cases diagnosed in 2012, and the sixth most common cause of death from cancer, with 400,169 deaths reported in 2012 (1). Squamous cell carcinoma (SCC) is by far the most common form of EC in Asian countries; adenocarcinomas of the lower third of the esophagus are common in western countries but account for only $4.3 \%$ of ECs in Japan (2). Surgery is considered the treatment of choice for patients with locoregionally confined EC. However, EC has a high rate of disease recurrence, even after curative surgery (3-6). Trimodality therapy, consisting of preoperative chemoradiation followed by esophagectomy, was developed to improve the survival of patients with locoregional $\mathrm{EC}$, and has become the standard treatment in the NCCN guidelines for patients with resectable esophageal SCC (7). In Japan, the standard treatment recommended by the Japan Clinical Oncology Group (JCOG) 9907 Study for patients with UICC (Union for International Cancer Control) stage II/III resectable $\mathrm{SCC}$ is a preoperative chemotherapy regimen of 5-fluorouracil (5-FU) and cisplatin (CP; the regimen: $\mathrm{CF}$ ) followed by esophagectomy (8). In that randomized phase III trial, patients in the neoadjuvant chemotherapy (NAC) +surgery group had a better survival rate than patients in the postoperative adjuvant group. However, the study was limited by its exclusion of patients with pathological NO disease from the adjuvant chemotherapy group and the disparity of patients who received postoperative chemotherapy (64.6\%) and preoperative chemotherapy (97.0\%). Additionally, a subset analysis of the JCOG 9907 study showed that NAC was not effective in patients with stage III disease or T3 tumors, thus demonstrating the need for more effective perioperative 
intervention in these patients. To improve survival, beginning in 1996, we introduced preoperative chemoradiotherapy (CRT) for patients with resectable advanced EC. A subsequent analysis showed increased tumor resectability, lower incidences of both local recurrence and distant metastasis, and a more favorable prognosis for CRT responders $(9,10)$. Additionally, a comparison of survival by patients with stage II/III esophageal SCC treated with surgery alone vs. with a protocol including neoadjuvant CRT showed that neoadjuvant CRT with CF significantly improved disease-free survival (DFS) compared with surgery alone (11); but these were retrospective, observational studies that may have included some confounding biases. Propensity score (PS) methods, including inverse probability of treatment weighting (IPTW) and propensity score matching (PSM) are used increasingly to reduce or minimize confounding effects in evaluations of treatment, exposure, or intervention based on observational or non-randomized data (12). In our retrospective study of patients with resectable EC, we compared those who received neoadjuvant CRT plus surgery (trimodality therapy) with those treated by surgery alone. After applying IPTW and PSM, we investigated whether survival benefits were obtained for patients in the neoadjuvant CRT plus surgery group.

\section{Patients and methods}

Patients with biopsy-proven, invasive SCC of the esophagus that had not been previously treated were eligible for the study. Other inclusion criteria were endoscopy and CT scan and/or endoscopic ultrasound examination findings of resectable, clinical stage II, III, or IV disease based on the UICC TNM Classification of Malignant Tumors, 6th ed (13). Other eligibility criteria were as follows: $<80$ years old, adequate organ function $(\mathrm{WBC} \geq 3,500, \mathrm{Hb} \geq 10 \mathrm{~g} / \mathrm{dl}$, ALT/AST $\leq 2 x$ upper limit of normal, platelets $\geq 100,000$, serum creatinine $\leq 1.3$ ), and an Eastern Cooperative Oncology Group performance status of $<1$ at the time of admission. The patients who received chemotherapy, chemoradiotherapy or endocrine therapy due to other malignancies were excluded in this study. We defined T3.5 on CT scan as suspected T4, possibly down-staged following neoadjuvant therapy, and included as T3. Of the 112 patients entered into this study, 55 received preoperative CRT followed by esophagectomy (Group A) and 57 underwent esophagectomy alone (Group B) between August 1996 and June 2015 at the Department of Surgery, Hyogo College of Medicine and Nara Hospital, Kindai University School of Medicine, Japan. Most patients of Group A were treated in Hyogo College of Medicine between 1996 to 2007, and most patients of Group B were treated in Nara Hospital between 2002 to 2015. All patients gave informed consent. This study was approved by the Ethical Committees of both Nata Hospital (No. 377), Kindai University School of Medicine (on the Kindai University web site); and Hyogo College of Medicine (No. 2906; on their web site).

Neoadjuvant CRT followed by esophagectomy. Preoperative radiotherapy was performed for 5 days per week (Monday to Friday, 2 Gy/day) using a linear accelerator (Mevatron KD2: Siemens, Germany). The radiation field encompassed the primary tumor volume (as defined by endoscopy, esophagography, and CT scan) with a $3-\mathrm{cm}$ margin in the cephalad and caudal directions and $4-\mathrm{cm}$ horizontal margins. If lymph node metastasis was detected by CT scan, the radiation field was extended to include the primary tumor and metastatic lesions. Twenty fractions of $2 \mathrm{~Gy}$ each were delivered for a total of 40 Gy of radiation per patient. Concurrent chemotherapy consisted of 5 -FU (500 mg/m²/day) administered for $120 \mathrm{~h}$ via continuous intravenous infusion starting on Day 1 and $\mathrm{CP}$ (15-20 mg/day) administered as a 2-h intravenous infusion on Days 1-5, repeated after 3 weeks.

Response to CRT was defined according to the criteria of RECIST guideline (version 1.1) (14). Esophagectomy was planned for 4-7 weeks after the completion of CRT. Most patients underwent thoracotomy, laparotomy, and cervicotomy in conjunction with the esophagectomy and two- or three-field lymphadenectomy. Reconstruction always consisted of a gastric tube and gastroesophageal anastomosis at the left side of the neck using a retrosternal root. Radical resection (R0) was defined as the removal of all macroscopic tumors with no evidence of distant metastasis, the absence of microscopic residual tumor, free resection margins, and lymphadenectomy extending beyond the involved nodes. Resection was defined as non-radical when microscopic (R1) or macroscopic (R2) residual tumor was found, according to the TNM criteria (13).

Esophagectomy for surgery alone. Esophagectomy was performed together with a small $(\sim 10 \mathrm{~cm})$ thoracotomy via thoracoscopy-assisted esophagectomy, with two- or three-field lymphadenectomy including the upper mediastinum. Reconstruction was routinely performed using a retrosternal root and a gastroesophageal anastomosis at the left side of the neck. The degree of radical resection (R) was assessed according to the TNM system (13).

Statistical analysis. The differences between the characteristics of patients in Group A and Group B were analyzed using the chi-square, Fisher's exact test and Mann-Whitney tests. Overall survival (OS) was defined as the time from the date of initial treatment to patient death or the date of the last available information on the patient's vital status. DFS was defined as the period after treatment during which time no cancer was found. With the unmatched data, differences between the cumulative survival rates in the two groups of patients were calculated using the log-rank test for comparison, as well as Kaplan-Meier survival curves and unadjusted Cox proportional hazard models. $\mathrm{P}<0.05$ was considered significant. Because therapy was not randomly assigned in this patient population, potential confounding and selection biases were accounted for by developing a PS, calculated using a multivariable logistic regression model in which age, sex, cancer site (location), primary tumor length, cTNM stage, lymph node metastasis, and depth of tumor invasion (cT) were the independent variables. After propensity scores were calculated, distributions of propensity scores and it after IPTW in two evaluated groups were analyzed by Kernel density estimation. Initially, the PSs were used to match preoperative CRT patients to those treated by surgery alone according to caliper matching in propensity score analysis. Next, the PSs were used to match preoperative CRT patients to those treated by surgery alone according to 
Table I. Patients characteristics in the present study $(n=112)$.

\begin{tabular}{|c|c|c|c|}
\hline Variable & Preoperative CRT (group A) & Surgery alone (group B) & P-value \\
\hline No. of patients & 55 & 57 & \\
\hline Age (years) & 60.49 & 65.19 & $\mathrm{P}<0.05$ \\
\hline Male/female & $44 / 10$ & $48 / 9$ & NS \\
\hline Location of primary tumor & & & $\mathrm{P}<0.05$ \\
\hline Cervical & 1 & 0 & \\
\hline Upper thoracic & 6 & 3 & \\
\hline Middle thoracic & 32 & 23 & \\
\hline Lower thoracic & 15 & 30 & \\
\hline Abdominal & 1 & 1 & \\
\hline T-classification (cT) & & & NS \\
\hline $\mathrm{cT} 2$ & 7 & 10 & \\
\hline cT3 (T3.5 included) & 45 & 46 & \\
\hline cT4 & 3 & 1 & \\
\hline Tumor length & 6.76 & 5.05 & $\mathrm{P}<0.05$ \\
\hline Clinical stage (TNM 6th edition) & & & NS \\
\hline II & 28 & 28 & \\
\hline III & 21 & 26 & \\
\hline IV & 7 & 3 & \\
\hline N-classification (cN) & & & $\mathrm{P}<0.05$ \\
\hline No & 36 & 18 & \\
\hline N1 & 19 & 39 & \\
\hline Resectability & & & NS \\
\hline R0 & 40 & 50 & \\
\hline $\mathrm{R} 1$ & 15 & 6 & \\
\hline $\mathrm{R} 2$ & 0 & 1 & \\
\hline
\end{tabular}

NS, not significant; CRT, chemoradiotherapy.

the IPTW method. IPTW was calculated from following formula: [1/PS] in Group A and [1/(1-PS)] in Group B. After IPTW and PSM, prognosis of patients in the two groups were examined using univariate analyses for OS and DFS. Cox proportional hazard models for OS were then adjusted using the IPTW values of the two groups and subgroups. Statistical analyses were performed using STATA version13 (Stata Corp LP, College Station, TX, USA), R version 3.1.1 (R Project for Statistical Computing, Vienna, Austria) and JMP version 11 (SAS Institute Inc., Tokyo, Japan).

\section{Results}

Patients characteristics in the unmatched analysis. Characteristics of the 112 patients included in this study are summarized in Table I. Group A consisted of 55 patients treated with neoadjuvant CRT+surgery, and Group B of 57 patients who underwent surgery only. All tumors were histologically confirmed to be esophageal SCC. The median age in Group A was significantly younger than in Group B, and tumor locations were generally lower in Group B than in Group A. Group B had a higher rate of cN0 disease than Group A. Although the tumors of group A patients were longer than those of group B patients, the difference in the cT between the two groups was not significant. PS distributions between the two treatment groups are shown in Fig. 1, and shown in Fig. 2 after IPTW. The PS distribution differed between the two groups. After IPTW, PSs were similar between two groups. After PSs were calculated, 46 of 112 patients were matched by caliper-matching method (Table II). Group A and Group B did not significantly differ in each parameter.

Effects of chemoradiation for Group A. Clinical responses (complete responses +partial responses) to neoadjuvant CRT for primary tumors and metastatic nodes were noted in 45 of 55 patients (clinical response rate: $81.8 \%$ ). All patients received $\mathrm{R} 0$ or $\mathrm{R} 1$ resections (Table I).

Survival analysis. In the unadjusted data, OS was higher in Group A than in Group B patients, but the difference was not significant. The OS for Group A was $55.36 \%$ at 3 years and 53.06 at 5 years; in Group B, the corresponding value at both time points was $43.44 \%$ ( $P=0.138$, Fig. 3 ). DFS was significantly better in Group A than in Group B; in Group A, DFS at 3 and 5 years was $52.21 \%$, whereas in Group B it was $38.7 \%$ at 3 years and $35.63 \%$ at 5 years $(\mathrm{P}<0.05$, Fig. 3$)$. In Group A, 


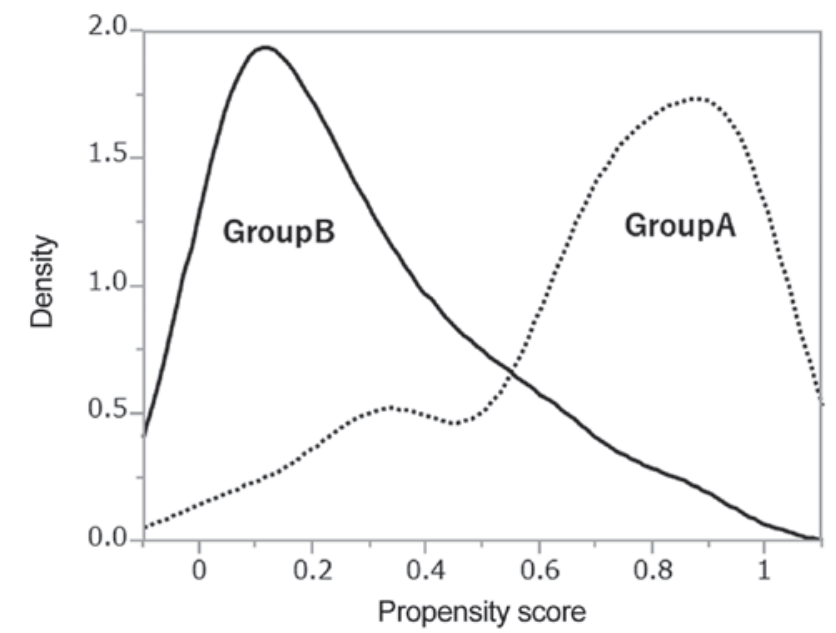

Figure 1. Different distributions of propensity scores between the two treatment groups.

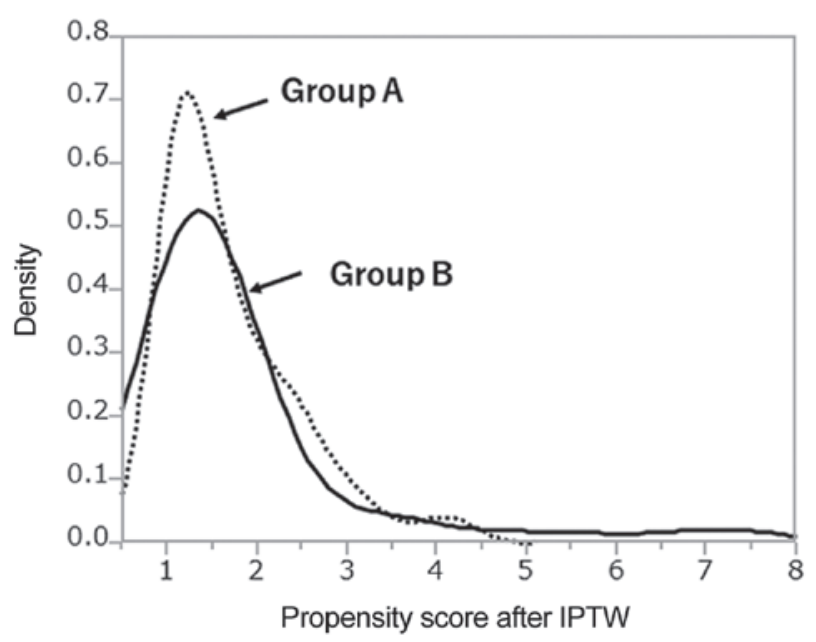

Figure 2. Distributions of propensity scores after IPTW between the two treatment groups. The distributions of both groups were similar according to the propensity score after IPTW. IPTW, inverse probability of treatment weighting.

5-year OS of patients with R0 resections was significantly higher $(72.93 \%)$ than in the $\mathrm{R} 1$ subgroup $(6.25 \%, \mathrm{P}<001)$. Also, 5 -year OS of patients who responded to CRT (63.01\%) was better than in the non-responder subgroup $(0 \%$; $\mathrm{P}<0.01)$.

After PSM, both OS and DFS were significantly higher in Group A than Group B (5-year OS: 65.2\% vs. 31.2\%, 5-year DFS: $65.2 \%$ vs. $20.9 \%$, P<0.05, Fig. 4). After IPTW was applied, OS was significantly higher in Group A than in Group B, with 3- and 5-year rates of 73.77 and $73.18 \%$, respectively, vs. $37.69 \%$ each [hazard ratio (HR): $0.2899,95 \%$ confidence interval (CI): 0.1167-0.7205, $\mathrm{P}<0.05$, Fig. 5]. DFS was also significantly higher in Group A than in Group B patients, with rates at 3 and 5 years of $72.38 \%$ each vs. 34.12 and $29.37 \%$ (HR: 0.2703, 95\% CI: 0.1123-0.6506, P<0.05, Fig. 5). Results of subgroup analyses of OS with respect to clinical lymph node status, cT, length, clinical stage, and location are shown in Fig. 6. Treatment was more effective in the preoperative CRT group with stage II disease that involved the mid-esophagus, clinical N0, T3 status, and patients age $>70$ years. Preoperative CRT was significantly effective regardless of tumor length.
Table II. Patients characteristics after propensity score matching in the present study $(n=46)$.

\begin{tabular}{|c|c|c|}
\hline Variable & $\begin{array}{c}\text { Preoperative } \\
\text { CRT } \\
\text { (group A) }\end{array}$ & $\begin{array}{l}\text { Surgery } \\
\text { alone } \\
\text { (group B) }\end{array}$ \\
\hline No. of patients & 23 & 23 \\
\hline Age (years) & 62.38 & 63.52 \\
\hline Male/female & $20 / 3$ & $19 / 4$ \\
\hline \multicolumn{3}{|c|}{ Location of primary tumor } \\
\hline Cervical & 1 & 0 \\
\hline Upper thoracic & 0 & 1 \\
\hline Middle thoracic & 12 & 11 \\
\hline Lower thoracic & 9 & 10 \\
\hline Abdominal & 1 & 1 \\
\hline \multicolumn{3}{|l|}{ T-classification (cT) } \\
\hline cT2 & 1 & 2 \\
\hline cT3 (T3.5 included) & 20 & 20 \\
\hline cT4 & 2 & 1 \\
\hline Tumor length & 6.15 & 6 \\
\hline \multirow{2}{*}{\multicolumn{3}{|c|}{$\begin{array}{l}\text { Clinical stage } \\
\text { (TNM 6th edition) }\end{array}$}} \\
\hline & & \\
\hline II & 9 & 12 \\
\hline III & 12 & 9 \\
\hline IV & 2 & 2 \\
\hline \multicolumn{3}{|l|}{ N-classification (cN) } \\
\hline No & 8 & 10 \\
\hline N1 & 15 & 13 \\
\hline \multicolumn{3}{|l|}{ Resectability } \\
\hline R0 & 18 & 21 \\
\hline $\mathrm{R} 1$ & 5 & 2 \\
\hline
\end{tabular}

CRT, chemoradiotherapy.

\section{Discussion}

This study was carried out to evaluate the clinical significance of preoperative CRT compared with surgery alone in patients with resectable esophageal SCC. After applying IPTW and PSM using PS, our results showed that preoperative CRT yielded superior survival rates compared with surgery alone.

PS may be used four ways to reduce confounding: PS matching, PS stratification, applying IPTW along with PS, and covariate adjustment using PS $(15,16)$. Several studies have examined the performance of these different PS methods for estimating treatment effects when the outcomes are binary (17-20). Austin reported that PS matching and application of the IPTW allow estimation of marginal HRs with minimal bias. The study concluded that IPTW gave estimates with a lower mean squared error when estimating the effect of treatment (12). We therefore evaluated the survival benefit of preoperative CRT vs. surgery alone after applying both PS matching and IPTW. Four reports employed a prognostic analysis using IPTW to analyze the 

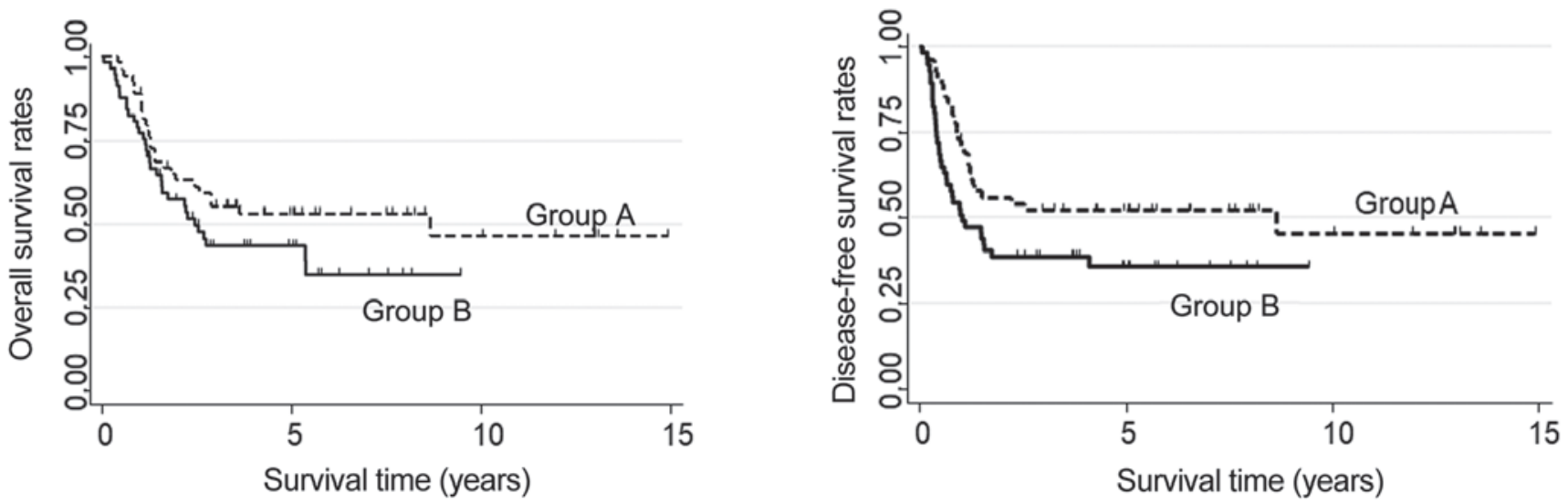

Figure 3. Overall survival and disease-free survival of unmatched patients treated with neoadjuvant chemoradiotherapy plus surgery (Group A) vs. surgery alone (Group B). They did not significantly differ in overall survival, but disease-free survival was significantly higher in Group A than in Group B (P<0.05).
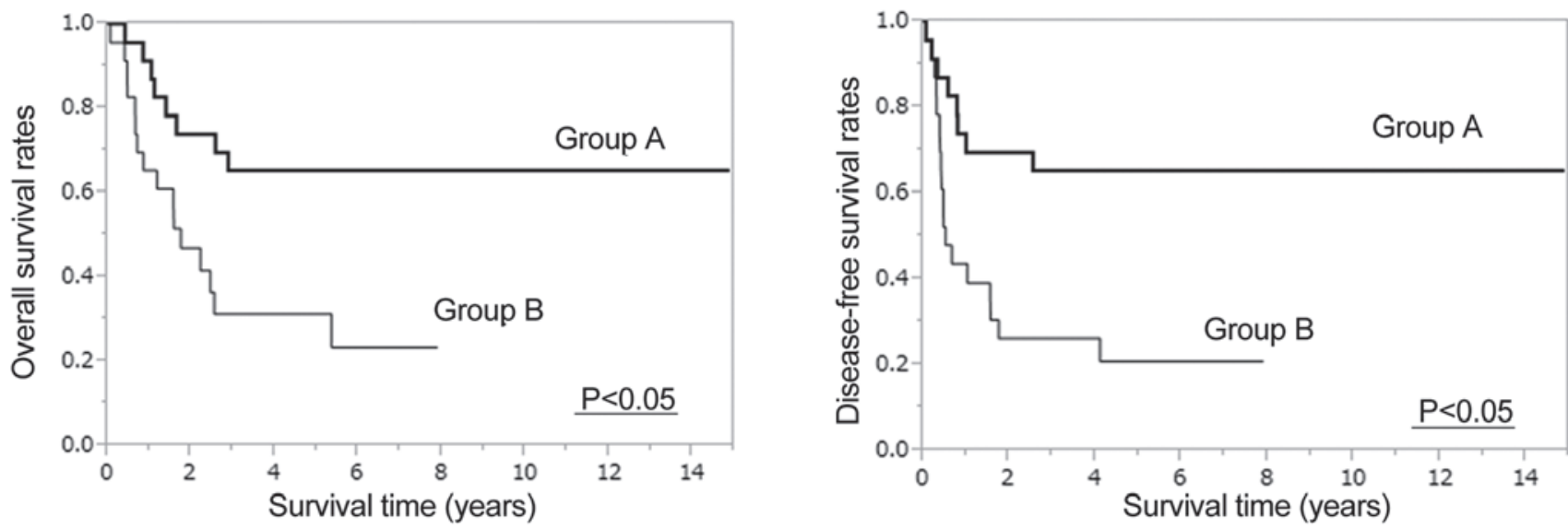

Figure 4. Overall survival and disease-free survival after propensity score matching were significantly higher in Group A than Group B (P<0.05).
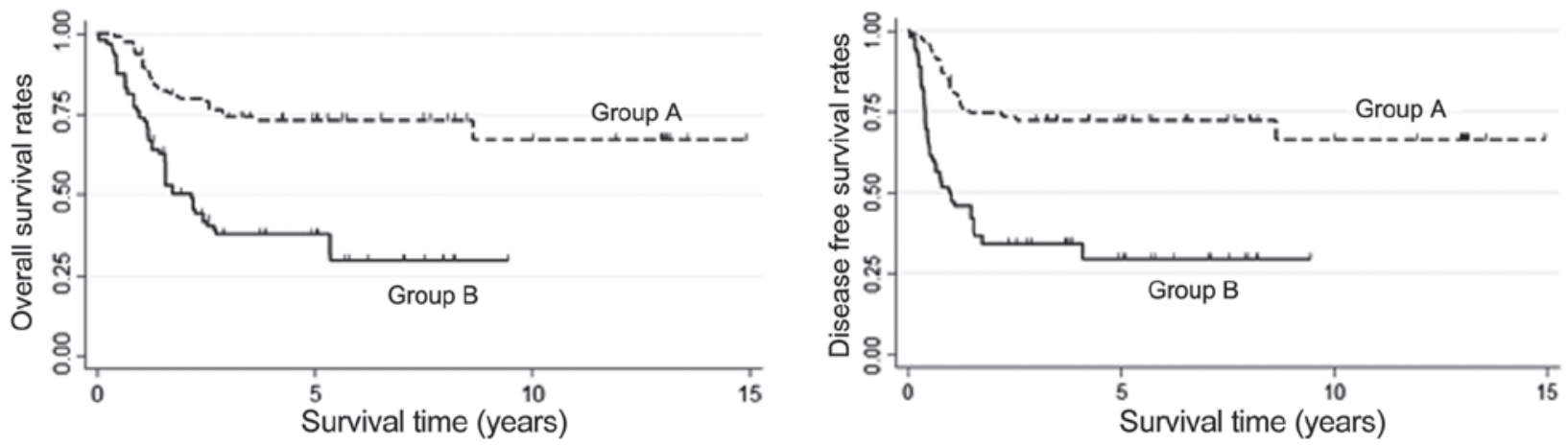

Figure 5. Overall survival and disease-free survival (DFS) of Groups A and B patients after analyzing data using inverse probability of treatment weighting. Overall survival and disease-free survival were significantly higher in Group A than in Group B $(\mathrm{P}<0.05)$.

data of patients with gastric and EC (21-24). Two of those reports used PS matching and IPTW to evaluate the effects of perioperative chemotherapy in patients with gastroesophageal adenocarcinoma $(23,24)$. Fiteni et al reported better outcomes with perioperative chemotherapy [docetaxel, cisplatin, and fluorouracil (DCF) regimen] than with surgery alone, after applying both IPTW and PS matching to the analysis. However, Kim et al compared standard CF therapy with DCF therapy and obtained better outcomes from DCF, using IPTW but not
PS matching. The authors explained the different results by the smaller sample size in the PS matched group, noting that when the sample size is small, IPTW is more reliable than PS matching. We used these two methods to analyze survival after esophagectomy. Fortunately, the same results were obtained in two analytical methods.

As shown in Fig. 1, the density of propensity scores had different distributions in both groups; therefore the number of matched patients had decreased. Because only 46/112 (41.1\%) 


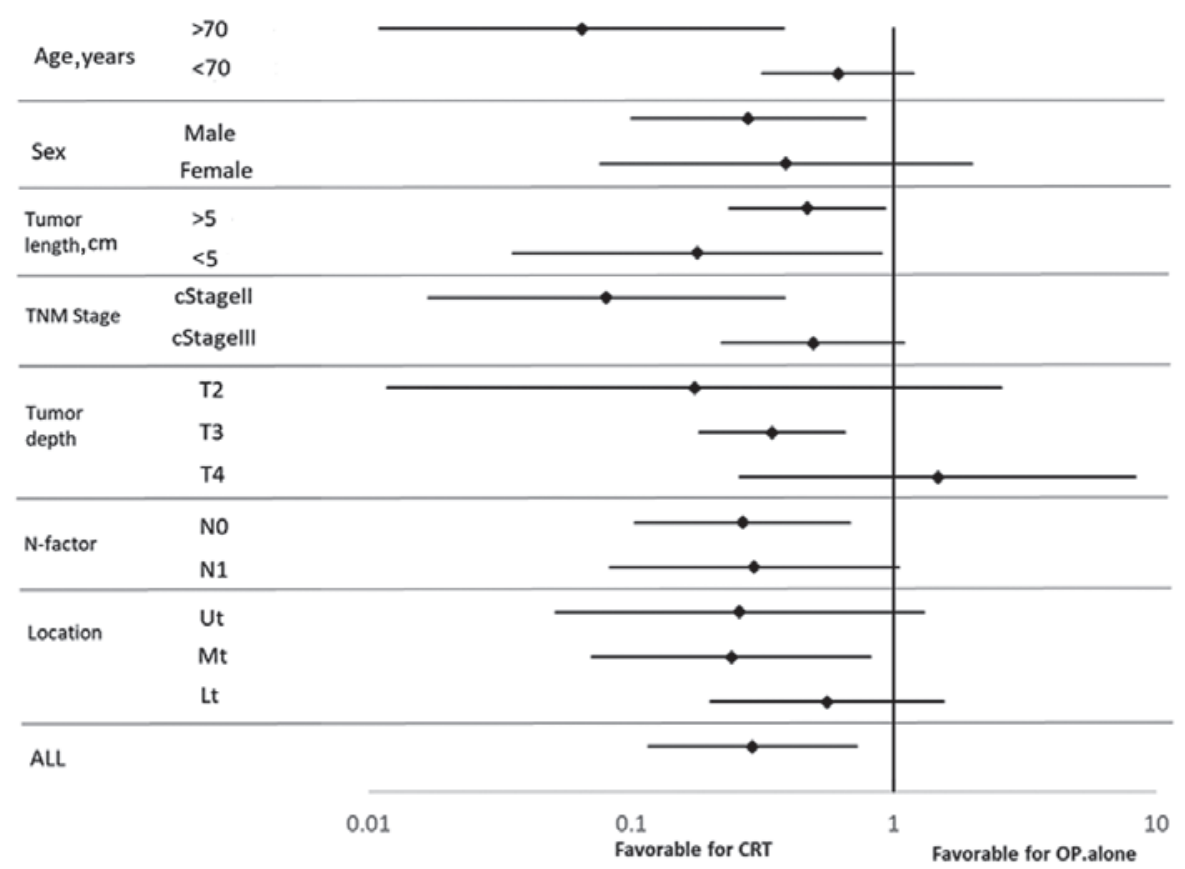

Figure 6. Forest plot of treatment effects on clinical factors for all patients. Ut, upper third of esophagus; Mt, middle third of esophagus; Lt, lower third of esophagus.

of patients were matched in the present study, statistical errors might have occurred. However, distributions of PS after IPTW were similar in both evaluated groups as shown in Fig. 2. The IPTW is not a method of increasing the number of patients, but rather a method to estimate a large amount of information from little information without changing the number of patients. When the number of patients is small, it might be necessary to evaluated both PS matching and IPTW. Two other reports assessed only patients with EC, and used only the IPTW method $(21,22)$. In the study of Guttmann et al, outcomes of elderly patients with EC treated with preoperative CRT vs. surgery alone was examined using the IPTW method, with better outcome noted in the preoperative CRT group. Also, Yendamuri et al reported that IPTW is preferred to matching, and other methods, in the context of a time-to-event analysis to estimate the average treatment effect. They evaluated the clinical characteristics of esophageal adenosquamous cell carcinoma, and compared adenocarcinoma and SCC. As adenosquamous cell carcinoma makes up only $1 \%(n=284)$ of 28,110 EC cases in the SEER database, therefore, it is presumed that they preferred only IPTW metho. Based on these four papers, in our evaluation of the two types of treatment, IPTW seemed to be the better method of analysis rather than PSM, especially it is considered to be excellent analysis for PS when the number of patients is small.

In Japan the standard treatment of resectable EC is preoperative chemotherapy using $5-\mathrm{FU}+\mathrm{CP}$, established in the JCOG 9907 study (8). However, in the NCCN guidelines, the standard treatment of SCC staged as T1b-T4a, N0-N+ or M0 is preoperative chemoradiation followed by esophagectomy (7). The preferred CRT regimens are paclitaxel/carboplatin, 5-FU/CP or 5-FU/oxaliplatin, and the recommended irradiation dose is 41.4-50.4 Gy.

In the ESMO guideline, standard treatment of local/locoregional (M0) SCC is neoadjuvant chemoradiation followed by esophagectomy (25). This guideline for EC was based on a meta-analysis and the results of the CROSS trial (26-29), in which patients were treated with carboplatin, paclitaxel, and 41.4 Gy of irradiation followed by surgery. The median survival of these patients was 81.6 months in the neoadjuvant CRT group and 21.1 months in the group treated with surgery alone; the difference was statistically significant. Despite the high survival rates reported with this regimen, our patients did not receive carboplatin because it has not been tested in a clinical trial in Japan. Clinical trials of this regimen should be planned to verify its efficacy in Japanese patients with EC.

Despite its long study period, the survival rates of our present study were similar to those of the JCOG 9907 study; therefore, we believe our present survival rates warrant evaluation. As no randomized clinical report of preoperative CRT vs. surgery alone in Japan is available, quasi-randomization using propensity scores, as we have done here, are clinically useful.

Subgroup analysis after IPTW also showed that preoperative CRT was more effective in patients with the following characteristics: $>70$ years of age, male sex, clinical stage II, T3 disease, mid-esophageal tumor, and clinical N0 disease. Notably, preoperative CRT was more effective in treating T3 disease in the present study cohort that in those included in the subgroup analysis of the JCOG9907 report (8), which further supports preoperative CRT in the treatment of locoregional T3 disease. Although few papers report the effects of preoperative chemotherapy or CRT by tumor locations, the CF regimen might be especially effective for SCC of the mid esophagus $(8,30)$. The CROSS TRIAL results indicated that CRT was more effective for clinical N0 disease; similar results were obtained in this study. Additionally, preoperative CRT seemed to be more effective for older patients in our study; however, we cannot explain the reason for this result, as conflicting data also exist (30). 
In Japan, JCOG 1109 has been planned to verify the effectiveness of preoperative CRT in patients with stage II/III esophageal SCC. JCOG 1109 has three arms and is a randomized phase III study to compare preoperative chemotherapy with $\mathrm{CF}$, preoperative $\mathrm{CRT}$, and preoperative $\mathrm{DCF}$ chemotherapy. The results of this study are upcoming as of this writing.

In conclusion, after applying IPTW using the PS and PSM, we found that preoperative CRT plus esophagectomy led to superior survival than surgery alone in patients with esophageal SCC. Until the results of the JCOG1109 are available, our study supports the benefit of neoadjuvant CRT for EC patients, including those with T3 or higher disease. We also showed that IPTW using PS is an important statistical method in studies based on retrospective data derived from actual clinical practice.

\section{Acknowledgements}

The authors would like to thank Mrs. Kaku, medical assistant at Kindai University Hospital, for her assistance with data collection. The abstract was presented at American Society of Clinical Oncology (ASCO) 2016 annual meeting, June 3-7 2016 in Chicago and published as abstract no. e15532 in Journal of Clinical Oncology 34 (no. 15_Suppl): 2016.

\section{Funding}

No funding was received.

\section{Availability of data and materials}

All data and materials in the present study were anonymized. All data generated or analyzed during this study are included in this published article.

\section{Authors' contributions}

YF, SF, MT, MY, MI and KK collaborated in the study design, YF and SF collected and analyzed the data. YF, TU and MT also performed data analysis. MT was a major contributor in statistical analysis. AT, HK and $\mathrm{MH}$ constructed the figures. The tables of this article were constructed by YF, MO, HK and YW. YF, MH, HK and YW drafted the manuscript.

\section{Ethics approval and consent to participate}

All study participants provided their informed consent. The study design was approved by an ethics review board (authorization no. 377, Nata Hospital, Kinki University School of Medicine, 2906, Hyogo College of Medicine). Our study conforms to the guidelines set forth in the Helsinki Declaration of 1975, as revised in 2000 (5), concerning Human and Animal Rights, and the policy concerning informed consent was followed.

\section{Patient consent for publication}

All patients consented to publication at the time of their surgeries.

\section{Competing interests}

The authors declare that they have no competing interests.

\section{References}

1. Roth JA and Putnam JB Jr: Surgery for cancer of the esophagus. Semin Oncol 21: 453-461, 1994.

2. Tachimori Y, Ozawa S, Numasaki H, Fujishiro M, Matsubara H, Oyama T, Shinoda M, Toh Y, Udagawa H and Uno T; Registration Committee for Esophageal Cancer of the Japan Esophageal Society: Comprehensive registry of esophageal cancer in Japan, 2009. Esophagus 13: 110-137, 2016.

3. Dresner SM and Griffin SM: Pattern of recurrence following radical oesophagectomy with two-field lymphadenectomy. Br J Surg 87: 1426-1433, 2000.

4. Hulscher JB, van Sandick JW, Tijssen JG, Obertop H and van Lanschot JJ: The recurrence pattern of esophageal carcinoma after transhiatal resection. J Am Coll Surg 191: 143-148, 2000.

5. Nakagawa S, Kanda T, Kosugi S, Ohashi M, Suzuki T and Hatakeyama K: Recurrence pattern of squamous cell carcinoma of the thoracic esophagus after extended radical esophagectomy with three-field lymphadenectomy. J Am Coll Surg 198: 205-211, 2004.

6. Isono K, Sato H and Nakayama K: Results of a nationwide study on the three-field lymph node dissection of esophageal cancer. Oncology 48: 411-420, 1991.

7. Ajani JA and D'Amico TA: Esophageal and Esophagogastric Junction Cancers in NCCN Clinical Practice Guidelines in Oncology (NCCN Guidelines version 1.2018). https://www. nccn.org/professionals/physician_gls/pdf/esophageal.pdf.

8. Ando N, Kato H, Igaki H, Shinoda M, Ozawa S, Shimizu H, Nakamura T, Yabusaki H, Aoyama N, Kurita A, et al: A randomized trial comparing postoperative adjuvant chemotherapy with cisplatin and 5-fluorouracil versus preoperative chemotherapy for localized advanced squamous cell carcinoma of the thoracic esophagus (JCOG9907). Ann Surg Oncol 19: 68-74, 2012.

9. Fujiwara Y, Kamikonya N, Inoue T, Koishi K, Yoshikawa R, Nakao K, Yagyu R, Nishiwaki M, Fujiwara M, Kojima S, et al: Chemoradiotherapy for T3 and T4 squamous cell carcinoma of the esophagus using low-dose FP and radiation: A preliminary report. Oncol Rep 14: 1177-1182, 2005.

10. Fujiwara Y, Yoshikawa R, Kamikonya N, Nakayama T, Kitani K, Tsujie M, Yukawa M, Inoue $M$ and Yamamura T: Trimodality therapy of esophagectomy plus neoadjuvant chemoradiotherapy improves the survival of clinical stage II/III esophageal squamous cell carcinoma patients. Oncol Rep 28: 446-452, 2012.

11. Fujiwara Y, Yoshikawa R, Kamikonya N, Nakayama T, Kitani K, Tsujie M, Yukawa M, Hara J, Yamamura T and Inoue M: Neoadjuvant chemoradiotherapy followed by esophagectomy vs. surgery alone in the treatment of resectable esophageal squamous cell carcinoma. Mol Clin Oncol 1: 773-779, 2013.

12. Austin PC: The performance of different propensity score methods for estimating marginal hazard ratios. Stat Med 32: 2837-2849, 2013.

13. Sobin LH and Wittekind C: International Union Against Cancer (UICC): TNM Classification of Malignant Tumors. 6th edition. Wiley-Liss, New York, 2002.

14. Eisenhauer EA, Therasse P, Bogaerts J, Schwartz LH, Sargent D, Ford R, Dancey J, Arbuck S, Gwyther S, Mooney M, et al: New response evaluation criteria in solid tumours: Revised RECIST guideline (version 1.1). Eur J Cancer 45: 228-247, 2009.

15. Weitzen S, Lapane KL, Toledano AY, Hume AL and Mor V: Principles for modeling propensity scores in medical research: A systematic literature review. Pharmacoepidemiol Drug Saf 13: 841-853, 2004.

16. Austin PC: A critical appraisal of propensity-score matching in the medical literature between 1996 and 2003. Stat Med 27: 2037-2049, 2008.

17. Austin PC, Grootendorst P, Normand SL and Anderson GM: Conditioning on the propensity score can result in biased estimation of common measures of treatment effect: A monte carlo study. Stat Med 26: 754-768, 2007.

18. Austin PC: The performance of different propensity-score methods for estimating relative risks. J Clin Epidemiol 61: $537-545,2008$. 
19. Austin PC: The performance of different propensity-score methods for estimating differences in proportions (risk differences or absolute risk reductions) in observational studies. Stat Med 29: 2137-2148, 2010.

20. Austin PC: The performance of different propensity score methods for estimating marginal odds ratios. Stat Med 26: 3078-3094, 2007.

21. Guttmann DM, Mitra N, Metz JM, Plastaras J, Feng W and Swisher-McClure S: Neoadjuvant chemoradiation is associated with improved overall survival in older patients with esophageal cancer. J Geriatr Oncol 9: 40-46, 2018.

22. Yendamuri S, Malhotra U, Hennon M, Miller A, Groman A, Halloon A and Reid ME: Clinical characteristics of adenosquamous esophageal carcinoma. J Gastrointest Oncol 8: 89-95, 2017.

23. Fiteni F, Paget-Bailly S, Messager M, N'Guyen T, Lakkis Z Mathieu P, Lamfichekh N, Picard A, Benzidane B, Cléau D, et al: Docetaxel, Cisplatin, and 5-Fluorouracil as perioperative chemotherapy compared with surgery alone for resectable gastroesophageal adenocarcinoma. Cancer Med 5: 3085-3093, 2016.

24. Kim S, Paget-Bailly S, Messager M, Nguyen T, Mathieu P, Lamfichekh N, Fein F, Fratté S, Cléau D, Lakkis Z, et al: Perioperative docetaxel, cisplatin, and 5-fluorouracil compared to standard chemotherapy for resectable gastroesophageal adenocarcinoma. Eur J Surg Oncol 43: 218-225, 2017.

25. Lordick F, Mariette C, Haustermans K, Obermannová R and Arnold D; ESMO guidelines committee: oesophageal cancer: ESMO clinical practice guidelines for diagnosis, treatment and follow-up. Ann Oncol 27 (Suppl 5): v50-v57, 2016.

26. Kranzfelder M, Schuster T, Geinitz H, Friess H and Büchler P: Meta-analysis of neoadjuvant treatment modalities and definitive non-surgical therapy for oesophageal squamous cell cancer Br J Surg 98: 768-783, 2011.
27. Sjoquist KM, Burmeister BH, Smithers BM, Zalcberg JR, Simes RJ, Barbour A and Gebski V; Australasian Gastro-Intestinal Trials Group: Survival after neoadjuvant chemotherapy or chemoradiotherapy for resectable oesophageal carcinoma: An updated meta-analysis. Lancet Oncol 12: 681-692, 2011.

28. Markar SR, Karthikesalingam A, Thrumurthy S and Low DE: Volume-outcome relationship in surgery for esophageal malignancy: Systematic review and meta-analysis 2000-2011. J Gastrointestinal Surg 16: 1055-1063, 2012.

29. Shapiro J, van Lanschot JJB, Hulshof MCCM, van Hagen P, van Berge Henegouwen MI, Wijnhoven BPL, van Laarhoven HWM, Nieuwenhuijzen GAP, Hospers GAP, Bonenkamp JJ, et al: CROSS study group: Neoadjuvant chemoradiotherapy plus surgery versus surgery alone for oesophageal or junctional cancer (CROSS): Long-term results of a randomised controlled trial. Lancet Oncol 16: 1090-1098, 2015.

30. Burmeister BH, Smithers BM, Gebski V, Fitzgerald L, Simes RJ, Devitt P, Ackland S, Gotley DC, Joseph D, Millar J, et al: Surgery alone versus chemoradiotherapy followed by surgery for resectable cancer of the oesophagus: A randomised controlled phase III trial. Lancet Oncol 6: 659-668, 2005.

This work is licensed under a Creative Commons Attribution-NonCommercial-NoDerivatives 4.0 International (CC BY-NC-ND 4.0) License. 